\title{
Blow-up of solutions for a quasilinear system with degenerate damping terms
}

\author{
Salah Boulaaras ${ }^{1,2^{*}}$, Abdelbaki Choucha ${ }^{3}$, Praveen Agarwal ${ }^{4}$, Mohamed Abdalla $^{5,6}$ and Sahar Ahmed Idris $^{7}$
}

\section{"Correspondence:}

s.boularas@qu.edu.sa

${ }^{1}$ Department of Mathematics, College of Sciences and Arts, ArRas, Qassim University, Buraydah, Kingdom of Saudi Arabia

${ }^{2}$ Laboratory of Fundamental and Applied Mathematics of Oran (LMFAO), University of Oran 1, Oran, Algeria

Full list of author information is available at the end of the article

\begin{abstract}
In this work, we consider a quasilinear system of viscoelastic equations with degenerate damping and general source terms. According to some suitable hypothesis, we study the blow-up of solutions. This is the general case of the recent results of Boulaaras' works (Bull. Malays. Math. Sci. Soc. 43:725-755, 2020) and (Appl. Anal. 99:1724-1748, 2020).
\end{abstract}

MSC: 35B40; 35L45; 93D15; 93D20

Keywords: Viscoelastic equation; Blow-up; Strong damping; Distributed delay

\section{Introduction}

In this paper, we consider the following problem:

$$
\left\{\begin{array}{l}
\left|u_{t}\right|^{\eta} u_{t t}-M\left(\|\nabla u\|_{2}^{2}\right) \Delta u+\int_{0}^{t} h_{1}(t-s) \Delta u(s) d s-\Delta u_{t t}+\left(|u|^{k}+|v|^{l}\right)\left|u_{t}\right|^{j-1} u_{t} \\
\quad=f_{1}(u, v), \quad(x, t) \in \Omega \times(0, T), \\
\left|v_{t}\right|^{\eta} v_{t t}-M\left(\|\nabla v\|_{2}^{2}\right) \Delta v+\int_{0}^{t} h_{2}(t-s) \Delta v(s) d s-\Delta v_{t t}+\left(|v|^{\theta}+|u|^{\varrho}\right)\left|v_{t}\right|^{s-1} v_{t} \\
\quad=f_{2}(u, v), \quad(x, t) \in \Omega \times(0, T), \\
u(x, t)=v(x, t)=0, \quad(x, t) \in \partial \Omega \times(0, T), \\
u(x, 0)=u_{0}(x), \quad u_{t}(x, 0)=u_{1}(x), \quad x \in \Omega, \\
v(x, 0)=v_{0}(x), \quad v_{t}(x, 0)=v_{1}(x), \quad x \in \Omega,
\end{array}\right.
$$

where $k, l, \theta, \varrho \geq 0 ; j, s \geq 1$ for $N=1,2$, and $0 \leq j, s \leq \frac{N+2}{N-2}$ for $N \geq 3$; and $\eta \geq 0$ for $N=1,2$ and $0<\eta \leq \frac{2}{N-2}$ for $N \geq 3, h_{i}(\cdot): R^{+} \rightarrow R^{+}(i=1,2)$ are positive relaxation functions which will be specified later. $\left(|(\cdot)|^{a}+|(\cdot)|^{b}\right)\left|(\cdot)_{t}\right|^{\tau-1}(\cdot)_{t}$ and $-\Delta(\cdot)_{t t}$ are the degenerate damping term and the dispersion term, respectively, and $M(\sigma)$ is a nonnegative locally Lipschitz function for $\gamma, \sigma \geq 0$ like $M(\sigma)=\alpha_{1}+\alpha_{2} \sigma^{\gamma}$. Especially, we select $\alpha_{1}=\alpha_{2}=1$, and

$$
\left\{\begin{array}{l}
f_{1}(u, v)=a_{1}|u+v|^{2(p+1)}(u+v)+b_{1}|u|^{p} \cdot u \cdot|v|^{p+2} \\
f_{2}(u, v)=a_{1}|u+v|^{2(p+1)}(u+v)+b_{1}|v|^{p} \cdot v \cdot|u|^{p+2} .
\end{array}\right.
$$

(c) The Author(s) 2021. This article is licensed under a Creative Commons Attribution 4.0 International License, which permits use, sharing, adaptation, distribution and reproduction in any medium or format, as long as you give appropriate credit to the original author(s) and the source, provide a link to the Creative Commons licence, and indicate if changes were made. The images or other third party material in this article are included in the article's Creative Commons licence, unless indicated otherwise in a credit line to the material. If material is not included in the article's Creative Commons licence and your intended use is not permitted by statutory regulation or exceeds the permitted use, you will need to obtain permission directly from the copyright holder. To view a copy of this licence, visit http://creativecommons.org/licenses/by/4.0/. 
Physically, the relationship between the stress and strain history in the beam inspired by Boltzmann theory is called viscoelastic damping term, where the kernel of the term of memory is the function $h$ (for further details, see the references $[3,5-9,12,17,20]$ ). If $\eta \geq 0$, this type of problem has been studied by many authors. For more depth, here are some papers that focused on the study of this damping (for example, see $([10,11,13,16$, $19,25,27,28])$. The effect of the degenerate damping terms often appears in many applications and practical problems and turns a lot of systems into different problems worth studying. Recently, the stability, the asymptotic behavior, and blowing up of evolution systems with time degenerate damping have been studied by many authors, see $[1,2,18]$. The most important is the source term with nonlinear functions $f_{1}$ and $f_{2}$ satisfying appropriate conditions. In physics they appear in several issues and theories. Many researchers also touched on this type of problem in several different issues, where the global existence of solutions, stability, and blow-up of solutions were studied. For more information, the reader is referred to $[1,11,15,18,22-24,26]$.

Most recently, if $\gamma=0, \alpha_{1}=1$ our problem (1.1) was studied in [14]. Under some restrictions on the initial datum, standard conditions on relaxation functions, the authors established the global existence and proved the general decay of solutions. Based on all of the above results, we believe that the combination of these terms of damping (memory term, degenerate damping, dispersion, and the source terms) constitutes a new problem worthy of study and research, different from the above that we will try to shed light on. Our paper is divided into several sections: in the next section we lay down the hypotheses, concepts, and lemmas we need. In the last section we prove our main result.

\section{Preliminaries}

We prove the blow-up result under the following suitable assumptions.

(A1) $h_{i}: \mathbb{R}_{+} \rightarrow \mathbb{R}_{+}$are differentiable and decreasing functions such that

$$
h_{i}(t) \geq 0, \quad 1-\int_{0}^{\infty} h_{i}(s) d s=l_{i}>0, \quad i=1,2 .
$$

(A2) There exist constants $\xi_{1}, \xi_{2}>0$ such that

$$
h_{i}^{\prime}(t) \leq-\xi_{i} h_{i}(t), \quad t \geq 0, i=1,2 .
$$

Lemma 2.1 There exists a function $F(u, v)$ such that

$$
\begin{aligned}
F(u, v) & =\frac{1}{2(\rho+2)}\left[u f_{1}(u, v)+v f_{2}(u, v)\right] \\
& =\frac{1}{2(\rho+2)}\left[a_{1}|u+v|^{2(p+2)}+2 b_{1}|u v|^{p+2}\right] \geq 0,
\end{aligned}
$$

where

$$
\frac{\partial F}{\partial u}=f_{1}(u, v), \quad \frac{\partial F}{\partial v}=f_{2}(u, v),
$$

we take $a_{1}=b_{1}=1$ for convenience. 
Lemma 2.2 [22] There exist two positive constants $c_{0}$ and $c_{1}$ such that

$$
\frac{c_{0}}{2(\rho+2)}\left(|u|^{2(p+2)}+|v|^{2(p+2)}\right) \leq F(u, v) \leq \frac{c_{1}}{2(\rho+2)}\left(|u|^{2(\rho+2)}+|v|^{2(p+2)}\right) .
$$

Theorem 2.3 Assume that (2.1) and (2.2) hold. Let

$$
\begin{cases}-1<p<\frac{4-n}{n-2}, & n \geq 3 \\ p \geq-1, & n=1,2\end{cases}
$$

Then, for any initial data

$$
\left(u_{0}, u_{1}, v_{0}, v_{1}\right) \in \mathcal{H}
$$

problem (1.1) has a unique solution for some $T>0$

$$
\begin{aligned}
& u, v \in C\left([0, T] ; H^{2}(\Omega) \cap H_{0}^{1}(\Omega)\right), \\
& u_{t} \in C\left([0, T] ; H_{0}^{1}(\Omega)\right) \cap L^{j+1}(\Omega), \\
& v_{t} \in C\left([0, T] ; H_{0}^{1}(\Omega)\right) \cap L^{s+1}(\Omega),
\end{aligned}
$$

where

$$
\mathcal{H}=H_{0}^{1}(\Omega) \times L^{2}(\Omega) \times H_{0}^{1}(\Omega) \times L^{2}(\Omega) .
$$

Now, we define the energy functional.

Lemma 2.4 Assume that (2.1),(2.2), and (2.4) hold, let $(u, v)$ be a solution of $(1.1)$, then $E(t)$ is nonincreasing, that is,

$$
\begin{aligned}
E(t)= & \frac{1}{\eta+2}\left[\left\|u_{t}\right\|_{\eta+2}^{\eta+2}+\left\|v_{t}\right\|_{\eta+2}^{\eta+2}\right]+\frac{1}{2}\left[\left\|\nabla u_{t}\right\|_{2}^{2}+\left\|\nabla v_{t}\right\|_{2}^{2}\right] \\
& +\frac{1}{2(\gamma+1)}\left[\|\nabla u\|_{2}^{2(\gamma+1)}+\|\nabla v\|_{2}^{2(\gamma+1)}\right] \\
& +\frac{1}{2}\left[\left(1-\int_{0}^{t} h_{1}(s) d s\right)\|\nabla u\|_{2}^{2}+\left(1-\int_{0}^{t} h_{2}(s) d s\right)\|\nabla v\|_{2}^{2}\right] \\
& +\frac{1}{2}\left[\left(h_{1} o \nabla u\right)(t)+\left(h_{2} o \nabla v\right)(t)\right]-\int_{\Omega} F(u, v) d x
\end{aligned}
$$

satisfies

$$
\begin{aligned}
E^{\prime}(t) \leq & \frac{1}{2}\left[\left(h_{1}^{\prime} o \nabla u\right)(t)+\left(h_{2}^{\prime} o \nabla v\right)\right](t)-\frac{1}{2}\left[h_{1}(t)\|\nabla u\|_{2}^{2}+h_{2}(t)\|\nabla v\|_{2}^{2}\right] \\
& -\int_{\Omega}\left(|u|^{k}+|v|^{l}\right)\left|u_{t}\right|^{j+1} d x-\int_{\Omega}\left(|v|^{\theta}+|u|^{\varrho}\right)\left|v_{t}\right|^{s+1} d x
\end{aligned}
$$$$
\leq 0 \text {. }
$$ 
Proof By multiplying $(1.1)_{1},(1.1)_{2}$ by $u_{t}$, $v_{t}$ and integrating over $\Omega$, we get

$$
\begin{aligned}
\frac{d}{d t}\left\{\frac{1}{\eta+2}\left\|u_{t}\right\|_{\eta+2}^{\eta+2}+\frac{1}{\eta+2}\left\|v_{t}\right\|_{\eta+2}^{\eta+2}+\frac{1}{2}\left\|\nabla u_{t}\right\|_{2}^{2}+\frac{1}{2}\left\|\nabla v_{t}\right\|_{2}^{2}\right. \\
\quad+\frac{1}{2(\gamma+1)}\left[\|\nabla u\|_{2}^{2(\gamma+1)}+\|\nabla v\|_{2}^{2(\gamma+1)}\right] \\
\quad+\frac{1}{2}\left(-1 \int_{0}^{t} h_{1}(s) d s\right)\|\nabla u\|_{2}^{2}+\frac{1}{2}\left(-1 \int_{0}^{t} h_{2}(s) d s\right)\|\nabla v\|_{2}^{2} \\
\left.\quad+\frac{1}{2}\left(h_{1} o \nabla u\right)(t)+\frac{1}{2}\left(h_{2} o \nabla v\right)(t)-\int_{\Omega} F(u, v) d x\right\} \\
=-\int_{\Omega}\left(|u|^{k}+|v|^{l}\right)\left|u_{t}\right|^{j+1} d x-\int_{\Omega}\left(|v|^{\theta}+|u|^{\varrho}\right)\left|v_{t}\right|^{s+1} d x \\
\quad+\frac{1}{2}\left(h_{1}^{\prime} o \nabla u\right)-\frac{1}{2} h_{1}(t)\|\nabla u\|_{2}^{2}+\frac{1}{2}\left(h_{2}^{\prime} o \nabla v\right)-\frac{1}{2} h_{2}(t)\|\nabla v\|_{2}^{2},
\end{aligned}
$$

we obtain (2.5) and (2.6).

\section{Blow-up}

In this section, we prove the blow-up result of solution of problem (1.1).

First, we define the functional

$$
\begin{aligned}
\mathbb{H}(t)=-E(t)= & -\frac{1}{\eta+2}\left[\left\|u_{t}\right\|_{\eta+2}^{\eta+2}+\left\|v_{t}\right\|_{\eta+2}^{\eta+2}\right]-\frac{1}{2}\left[\left\|\nabla u_{t}\right\|_{2}^{2}+\left\|\nabla v_{t}\right\|_{2}^{2}\right] \\
& -\frac{1}{2(\gamma+1)}\left[\|\nabla u\|_{2}^{2(\gamma+1)}+\|\nabla v\|_{2}^{2(\gamma+1)}\right] \\
& -\frac{1}{2}\left[\left(1-\int_{0}^{t} h_{1}(s) d s\right)\|\nabla u\|_{2}^{2}+\left(1-\int_{0}^{t} h_{2}(s) d s\right)\|\nabla v\|_{2}^{2}\right] \\
& -\frac{1}{2}\left[\left(h_{1} o \nabla u\right)(t)+\left(h_{2} o \nabla v\right)(t)\right] \\
& +\frac{1}{2(p+2)}\left[\|u+v\|_{2(p+2)}^{2(p+2)}+2\|u v\|_{p+2}^{p+2}\right] .
\end{aligned}
$$

Theorem 3.1 Assume that (2.1)-(2.2) and (2.4) hold, and suppose that $E(0)<0$ and

$$
2(p+2)>\max \{k+j+1 ; l+j+1 ; \theta+s+1 ; \varrho+s+1 ; 2(\gamma+1)\} \text {. }
$$

Then the solution of problem (1.1) blows up in finite time.

Proof From (2.5), we have

$$
E(t) \leq E(0) \leq 0
$$

Therefore

$$
\mathbb{H}^{\prime}(t)=-E^{\prime}(t) \geq \int_{\Omega}\left(|u|^{k}+|v|^{l}\right)\left|u_{t}\right|^{j+1} d x+\int_{\Omega}\left(|v|^{\theta}+|u|^{\varrho}\right)\left|v_{t}\right|^{s+1} d x
$$


Hence

$$
\begin{aligned}
& \mathbb{H}^{\prime}(t) \geq \int_{\Omega}\left(|u|^{k}+|v|^{l}\right)\left|u_{t}\right|^{j+1} d x \geq 0, \\
& \mathbb{H}^{\prime}(t) \geq \int_{\Omega}\left(|v|^{\theta}+|u|^{\varrho}\right)\left|v_{t}\right|^{s+1} d x \geq 0 .
\end{aligned}
$$

By (3.1) and (2.3), we have

$$
\begin{aligned}
0 & \leq \mathbb{H}(0) \leq \mathbb{H}(t) \leq \frac{1}{2(p+2)}\left(\|u+v\|_{2(p+2)}^{2(p+2)}+2\|u v\|_{p+2}^{p+2}\right) \\
& \leq \frac{c_{1}}{2(p+2)}\left(\|u\|_{2(p+2)}^{2(p+2)}+\|v\|_{2(p+2)}^{2(p+2)}\right) .
\end{aligned}
$$

We set

$$
\begin{aligned}
\mathcal{K}(t)= & \mathbb{H}^{1-\alpha}+\frac{\varepsilon}{\eta+1} \int_{\Omega}\left[u\left|u_{t}\right|^{\eta} u_{t}+v\left|v_{t}\right|^{\eta} v_{t}\right] d x \\
& +\varepsilon \int_{\Omega}\left[\nabla u_{t} \nabla u+\nabla v_{t} \nabla v\right] d x
\end{aligned}
$$

where $\varepsilon>0$ is to be assigned later, and we assume that

$$
\begin{aligned}
0<\alpha< & \min \left\{\left(1-\frac{1}{2(p+2)}-\frac{1}{\eta+2}\right), \frac{1+2 \gamma}{4(\gamma+1)}, \frac{2 p+3-(k+j)}{2 j(p+2)},\right. \\
& \left.\frac{2 p+3-(l+j)}{2 j(p+2)}, \frac{2 p+3-(\theta+s)}{2 s(p+2)}, \frac{2 p+3-(\varrho+s)}{2 s(p+2)}\right\}<1 .
\end{aligned}
$$

By multiplying $(1.1)_{1},(1.1)_{2}$ by $u, v$ and with a derivative of (3.7), we get

$$
\begin{aligned}
& \mathcal{K}^{\prime}(t)=(1-\alpha) \mathbb{H}^{-\alpha} \mathbb{H}^{\prime}(t)+\frac{\varepsilon}{\eta+1}\left(\left\|u_{t}\right\|_{\eta+2}^{\eta+2}+\left\|v_{t}\right\|_{\eta+2}^{\eta+2}\right)+\varepsilon\left(\left\|\nabla u_{t}\right\|_{2}^{2}+\left\|\nabla v_{t}\right\|_{2}^{2}\right) \\
& +\underbrace{\varepsilon \int_{\Omega} \nabla u \int_{0}^{t} g(t-s) \nabla u(s) d s d x}_{J_{1}}+\underbrace{\varepsilon \int_{\Omega} \nabla v \int_{0}^{t} h(t-s) \nabla v(s) d s d x}_{J_{2}} \\
& -\underbrace{\varepsilon \int_{\Omega}\left(|u|^{k}+|v|^{l}\right)\left|u_{t}\right|^{j-1} u_{t} \cdot u d x}_{J_{3}}-\underbrace{\varepsilon \int_{\Omega}\left(|v|^{\theta}+|u|^{\varrho}\right)\left|v_{t}\right|^{s-1} v_{t} \cdot v d x}_{J_{4}} \\
& -\varepsilon\left(\|\nabla u\|_{2}^{2}+\|\nabla v\|_{2}^{2}\right)-\varepsilon\left(\|\nabla u\|_{2}^{2(\gamma+1)}+\|\nabla v\|_{2}^{2(\gamma+1)}\right) \\
& +\underbrace{\varepsilon\left[\|u+v\|_{2(p+2)}^{2(p+2)}+2\|u v\|_{p+2}^{p+2}\right]}_{J_{5}} .
\end{aligned}
$$

We have

$$
\begin{aligned}
J_{1} & =\varepsilon \int_{0}^{t} h_{1}(t-s) d s \int_{\Omega} \nabla u \cdot(\nabla u(s)-\nabla u(t)) d x d s+\varepsilon \int_{0}^{t} h_{1}(s) d s\|\nabla u\|_{2}^{2} \\
& \geq \frac{\varepsilon}{2} \int_{0}^{t} h_{1}(s) d s\|\nabla u\|_{2}^{2}-\frac{\varepsilon}{2}\left(h_{1} o \nabla u\right) .
\end{aligned}
$$




$$
\begin{aligned}
J_{2} & =\varepsilon \int_{0}^{t} h_{2}(t-s) d s \int_{\Omega} \nabla v \cdot(\nabla v(s)-\nabla v(t)) d x d s+\varepsilon \int_{0}^{t} h_{2}(s) d s\|\nabla v\|_{2}^{2} \\
& \geq \frac{\varepsilon}{2} \int_{0}^{t} h_{2}(s) d s\|\nabla v\|_{2}^{2}-\frac{\varepsilon}{2}\left(h_{2} o \nabla v\right) .
\end{aligned}
$$

From (3.9), we find

$$
\begin{aligned}
\mathcal{K}^{\prime}(t) \geq & (1-\alpha) \mathbb{H}^{-\alpha} \mathbb{H}^{\prime}(t)+\frac{\varepsilon}{\eta+1}\left(\left\|u_{t}\right\|_{\eta+2}^{\eta+2}+\left\|v_{t}\right\|_{\eta+2}^{\eta+2}\right)+\varepsilon\left(\left\|\nabla u_{t}\right\|_{2}^{2}+\left\|\nabla v_{t}\right\|_{2}^{2}\right) \\
& -\varepsilon\left[\left(1-\frac{1}{2} \int_{0}^{t} h_{1}(s) d s\right)\|\nabla u\|_{2}^{2}+\left(1-\frac{1}{2} \int_{0}^{t} h_{2}(s) d s\right)\|\nabla v\|_{2}^{2}\right] \\
& -\frac{\varepsilon}{2}\left(h_{1} o \nabla u\right)-\frac{\varepsilon}{2}\left(h_{2} o \nabla v\right)-\varepsilon\left(\|\nabla u\|_{2}^{2(\gamma+1)}+\|\nabla v\|_{2}^{2(\gamma+1)}\right) \\
& -J_{3}-J_{4}+J_{5} .
\end{aligned}
$$

At this point, we use Young's inequality for $\delta>0$

$$
X Y \leq \frac{\delta^{\alpha} X^{\alpha}}{\alpha}+\frac{\delta^{-\beta} X^{\beta}}{\beta}, \quad \alpha, \beta>0, \frac{1}{\alpha}+\frac{1}{\beta}=1
$$

we get, for $\delta_{1}, \delta_{2}>0$,

$$
\begin{aligned}
\left.|u| u_{t}\right|^{j-1} u_{t} \mid & \leq \frac{\delta_{1}^{j+1}}{j+1}|u|^{j+1}+\frac{j}{j+1} \delta_{1}^{-\left(\frac{j+1}{j}\right)}\left|u_{t}\right|^{j+1} \\
\left.|v| v_{t}\right|^{s-1} v_{t} \mid & \leq \frac{\delta_{2}^{s+1}}{s+1}|v|^{s+1}+\frac{s}{s+1} \delta_{2}^{-\left(\frac{s+1}{s}\right)}\left|v_{t}\right|^{s+1} .
\end{aligned}
$$

Hence, we have

$$
\begin{aligned}
& J_{3} \leq \varepsilon \frac{\delta_{1}^{j+1}}{j+1} \int_{\Omega}\left(|u|^{k}+|v|^{l}\right)|u|^{j+1} d x+\varepsilon \frac{j \delta_{1}^{-\left(\frac{j+1}{j}\right)}}{j+1} \int_{\Omega}\left(|u|^{k}+|v|^{l}\right)\left|u_{t}\right|^{j+1} d x \\
& J_{4} \leq \varepsilon \frac{\delta_{2}^{s+1}}{s+1} \int_{\Omega}\left(|v|^{\theta}+|u|^{\varrho}\right)|v|^{s+1} d x+\varepsilon \frac{s \delta_{2}^{-\left(\frac{s+1}{s}\right)}}{s+1} \int_{\Omega}\left(|v|^{\theta}+|u|^{\varrho}\right)\left|v_{t}\right|^{s+1} d x .
\end{aligned}
$$

Therefore, using (3.5) and by setting $\delta_{1}, \delta_{1}$ so that

$$
\frac{j \delta_{1}^{-\left(\frac{j+1}{j}\right)}}{j+1}=\frac{\kappa \mathbb{H}^{-\alpha}(t)}{2}, \quad \frac{s \delta_{2}^{-\left(\frac{s+1}{s}\right)}}{s+1}=\frac{\kappa \mathbb{H}^{-\alpha}(t)}{2}
$$

substituting in (3.12), we get

$$
\begin{aligned}
\mathcal{K}^{\prime}(t) \geq & {[(1-\alpha)-\varepsilon \kappa] \mathbb{H}^{-\alpha} \mathbb{H}^{\prime}(t)+\frac{\varepsilon}{\eta+1}\left(\left\|u_{t}\right\|_{\eta+2}^{\eta+2}+\left\|v_{t}\right\|_{\eta+2}^{\eta+2}\right) } \\
& -\varepsilon\left[\left(1-\frac{1}{2} \int_{0}^{t} h_{1}(s) d s\right)\|\nabla u\|_{2}^{2}+\left(1-\frac{1}{2} \int_{0}^{t} h_{2}(s) d s\right)\|\nabla v\|_{2}^{2}\right] \\
& +\varepsilon\left(\left\|\nabla u_{t}\right\|_{2}^{2}+\left\|\nabla v_{t}\right\|_{2}^{2}\right)-\frac{\varepsilon}{2}\left(h_{1} o \nabla u\right)-\frac{\varepsilon}{2}\left(h_{2} o \nabla v\right) \\
& -\varepsilon C_{1}(\kappa) \mathbb{H}^{\alpha j}(t) \int_{\Omega}\left(|u|^{k}+|v|^{l}\right)|u|^{j+1} d x
\end{aligned}
$$




$$
\begin{aligned}
& -\varepsilon C_{2}(\kappa) \mathbb{H}^{\alpha s}(t) \int_{\Omega}\left(|v|^{\theta}+|u|^{\varrho}\right)|v|^{s+1} d x \\
& -\varepsilon\left(\|\nabla u\|_{2}^{2(\gamma+1)}+\|\nabla v\|_{2}^{2(\gamma+1)}\right)+J_{5},
\end{aligned}
$$

where

$$
C_{1}(\kappa):=\left(\frac{2 j}{\kappa(j+1)}\right)^{j+1} \frac{1}{j+1}, \quad C_{2}(\kappa):=\left(\frac{2 s}{\kappa(s+1)}\right)^{s+1} \frac{1}{s+1}
$$

we have

$$
\begin{aligned}
& \int_{\Omega}\left(|u|^{k}+|v|^{l}\right)|u|^{j+1} d x=\|u\|_{k+j+1}^{k+j+1}+\int_{\Omega}|v|^{l}|u|^{j+1} d x, \\
& \int_{\Omega}\left(|v|^{\theta}+|u|^{\varrho}\right)|v|^{s+1} d x=\|v\|_{\theta+s+1}^{\theta+s+1}+\int_{\Omega}|u|^{\varrho}|v|^{s+1} d x .
\end{aligned}
$$

By Young's inequality, we find for $\delta_{3}, \delta_{4}>0$

$$
\begin{aligned}
& \int_{\Omega}|v|^{l}|u|^{j+1} d x \leq \frac{l}{l+j+1} \delta_{3}^{\left(\frac{l+j+1}{l}\right)}\|v\|_{l+j+1}^{l+j+1}+\frac{j+1}{l+j+1} \delta_{3}^{-\left(\frac{l+j+1}{l}\right)}\|u\|_{l+j+1}^{l+j+1}, \\
& \int_{\Omega}|u|^{\varrho}|v|^{s+1} d x \leq \frac{\varrho}{\varrho+s+1} \delta_{4}^{\left(\frac{\varrho+s+1}{\varrho}\right)}\|u\|_{\varrho+s+1}^{\varrho+s+1}+\frac{s+1}{\varrho+s+1} \delta_{4}^{-\left(\frac{\varrho+s+1}{\varrho}\right)}\|v\|_{\varrho+s+1}^{\varrho+s+1} .
\end{aligned}
$$

Hence

$$
\begin{aligned}
\mathbb{H}^{\alpha j}(t) \int_{\Omega}\left(|u|^{k}+|v|^{l}\right)|u|^{j+1} d x \leq & \mathbb{H}^{\alpha j}(t)\|u\|_{k+j+1}^{k+j+1}+\frac{l \mathbb{H}^{\alpha j}(t)}{l+j+1} \delta_{3}^{\left(\frac{l+j+1}{l}\right)}\|v\|_{l+j+1}^{l+j+1} \\
& +\frac{(j+1) \mathbb{H}^{\alpha j}(t)}{l+j+1} \delta_{3}^{-\left(\frac{l+j+1}{l}\right)}\|u\|_{l+j+1}^{l+j+1}, \\
\mathbb{H}^{\alpha s}(t) \int_{\Omega}\left(|v|^{\theta}+|u|^{\varrho}\right)|v|^{s+1} d x \leq & \mathbb{H}^{\alpha s}(t)\|v\|_{\theta+s+1}^{\theta+s+1}+\frac{\varrho \mathbb{H}^{\alpha s}(t)}{\varrho+s+1} \delta_{4}^{\left(\frac{\varrho+s+1}{\varrho}\right)}\|u\|_{\varrho+s+1}^{\varrho+s+1} \\
& +\frac{(s+1) \mathbb{H}^{\alpha s}(t)}{\varrho+s+1} \delta_{4}^{-\left(\frac{\varrho+s+1}{\varrho}\right)}\|v\|_{\varrho+s+1}^{\varrho+s+1} .
\end{aligned}
$$

Since (2.4) holds, we obtain by using (3.6) and (3.8)

$$
\begin{aligned}
& \mathbb{H}^{\alpha j}(t)\|u\|_{k+j+1}^{k+j+1} \leq c_{1}\left(\|u\|_{2(p+2)}^{2 \alpha j(p+2)+k+j+1}+\|v\|_{2(p+2)}^{2 \alpha j(p+2)}\|u\|_{k+j+1}^{k+j+1}\right), \\
& \mathbb{H}^{\alpha j}(t)\|v\|_{k+j+1}^{k+j+1} \leq c_{2}\left(\|v\|_{2(p+2)}^{2 \alpha j(p+2)+k+j+1}+\|u\|_{2(p+2)}^{2 \alpha j(p+2)}\|v\|_{k+j+1}^{k+j+1}\right), \\
& \mathbb{H}^{\alpha s}(t)\|v\|_{\theta+s+1}^{\theta+s+1} \leq c_{3}\left(\|v\|_{2(p+2)}^{2 \alpha s(p+2)+\theta+s+1}+\|u\|_{2(p+2)}^{2 \alpha s(p+2)}\|v\|_{\theta+s+1}^{\theta+s+1}\right), \\
& \mathbb{H}^{\alpha s}(t)\|u\|_{\theta+s+1}^{\theta+s+1} \leq c_{4}\left(\|u\|_{2(p+2)}^{2 \alpha s(p+2)+\theta+s+1}+\|v\|_{2(p+2)}^{2 \alpha s(p+2)}\|u\|_{\theta+s+1}^{\theta+s+1}\right)
\end{aligned}
$$

for some positive constants $c_{i}, i=1, \ldots, 4$. By using (3.8) and the algebraic inequality

$$
B^{\varsigma} \leq(B+1) \leq\left(1+\frac{1}{b}\right)(B+b), \quad \forall B>0,0<\varsigma<1, b>0
$$


we have $\forall t>0$

$$
\begin{aligned}
& \|u\|_{2(p+2)}^{2 \alpha j(p+2)+k+j+1} \leq d\left(\|u\|_{2(p+2)}^{2(p+2)}+\mathbb{H}(0)\right) \leq d\left(\|u\|_{2(p+2)}^{2(p+2)}+\mathbb{H}(t)\right), \\
& \|v\|_{2(p+2)}^{2 \alpha j(p+2)+k+j+1} \leq d\left(\|v\|_{2(p+2)}^{2(p+2)}+\mathbb{H}(0)\right) \leq d\left(\|v\|_{2(p+2)}^{2(p+2)}+\mathbb{H}(t)\right), \\
& \|v\|_{2(p+2)}^{2 \alpha s(p+2)+\theta+s+1} \leq d\left(\|v\|_{2(p+2)}^{2(p+2)}+\mathbb{H}(0)\right) \leq d\left(\|v\|_{2(p+2)}^{2(p+2)}+\mathbb{H}(t)\right), \\
& \|u\|_{2(p+2)}^{2 \alpha s(p+2)+\theta+s+1} \leq d\left(\|u\|_{2(p+2)}^{2(p+2)}+\mathbb{H}(0)\right) \leq d\left(\|u\|_{2(p+2)}^{2(p+2)}+\mathbb{H}(t)\right),
\end{aligned}
$$

where $d=1+\frac{1}{\mathbb{H}(0)}$. Also, since

$$
(X+Y)^{\gamma} \leq C\left(X^{\gamma}+Y^{\gamma}\right), \quad X, Y>0, \gamma>0,
$$

we conclude

$$
\begin{gathered}
\|v\|_{2(p+2)}^{2 \alpha j(p+2)}\|u\|_{k+j+1}^{k+j+1} \leq c_{5}\left(\|v\|_{2(p+2)}^{2(p+2)}+\|u\|_{2(p+2)}^{2(p+2)}\right), \\
\|u\|_{2(p+2)}^{2 \alpha j(p+2)}\|v\|_{k+j+1}^{k+j+1} \leq c_{6}\left(\|u\|_{2(p+2)}^{2(p+2)}+\|v\|_{2(p+2)}^{2(p+2)}\right), \\
\|u\|_{2(p+2)}^{2 \alpha s(p+2)}\|v\|_{\theta+s+1}^{\theta+s+1} \leq c_{7}\left(\|v\|_{2(p+2)}^{2(p+2)}+\|u\|_{2(p+2)}^{2(p+2)}\right), \\
\|v\|_{2(p+2)}^{2 \alpha s(p+2)}\|u\|_{\theta+s+1}^{\theta+s+1} \leq c_{8}\left(\|v\|_{2(p+2)}^{2(p+2)}+\|u\|_{2(p+2)}^{2(p+2)}\right) .
\end{gathered}
$$

Substituting (3.23) and (3.25) in (3.21), we get

$$
\begin{aligned}
& \mathbb{H}^{\alpha j}(t)\|u\|_{k+j+1}^{k+j+1} \leq c_{9}\left(\|v\|_{2(p+2)}^{2(p+2)}+\|u\|_{2(p+2)}^{2(p+2)}+\mathbb{H}(t)\right), \\
& \mathbb{H}^{\alpha j}(t)\|v\|_{k+j+1}^{k+j+1} \leq c_{10}\left(\|v\|_{2(p+2)}^{2(p+2)}+\|u\|_{2(p+2)}^{2(p+2)}+\mathbb{H}(t)\right), \\
& \mathbb{H}^{\alpha s}(t)\|v\|_{\theta+s+1}^{\theta+s+1} \leq c_{11}\left(\|v\|_{2(p+2)}^{2(p+2)}+\|u\|_{2(p+2)}^{2(p+2)}+\mathbb{H}(t)\right), \\
& \mathbb{H}^{\alpha s}(t)\|u\|_{\theta+s+1}^{\theta+s+1} \leq c_{12}\left(\|v\|_{2(p+2)}^{2(p+2)}+\|u\|_{2(p+2)}^{2(p+2)}+\mathbb{H}(t)\right) .
\end{aligned}
$$

Hence, by fixed $\delta_{3}, \delta_{4}>0$, we get

$$
\begin{aligned}
& \mathbb{H}^{\alpha j}(t) \int_{\Omega}\left(|u|^{k}+|v|^{l}\right)|u|^{j+1} d x \\
& \quad \leq M_{1}\left(1+\frac{l \delta_{3}^{\left(\frac{l+j+1}{l}\right)}}{l+j+1}+\frac{(j+1) \delta_{3}^{-\left(\frac{l+j+1}{l}\right)}}{l+j+1}\right)\left(\|v\|_{2(p+2)}^{2(p+2)}+\|u\|_{2(p+2)}^{2(p+2)}+\mathbb{H}(t)\right), \\
& \mathbb{H}^{\alpha s}(t) \int_{\Omega}\left(|v|^{\theta}+|u|^{\varrho}\right)|v|^{s+1} d x \\
& \quad \leq M_{2}\left(1+\frac{\varrho \delta_{4}^{\left(\frac{\varrho+s+1}{\varrho}\right)}}{\varrho+s+1}+\frac{(s+1) \delta_{4}^{-\left(\frac{\varrho+s+1}{\varrho}\right)}}{\varrho+s+1}\right)\left(\|v\|_{2(p+2)}^{2(p+2)}+\|u\|_{2(p+2)}^{2(p+2)}+\mathbb{H}(t)\right)
\end{aligned}
$$

for some constants $M_{1}, M_{2}>0$.

Now, for $0<a<1$, from (3.1)

$$
\begin{aligned}
J_{5} & =\varepsilon\left[\|u+v\|_{2(p+2)}^{2(p+2)}+2\|u v\|_{p+2}^{p+2}\right] \\
& =\varepsilon a\left[\|u+v\|_{2(p+2)}^{2(p+2)}+2\|u v\|_{p+2}^{p+2}\right]
\end{aligned}
$$




$$
\begin{aligned}
& +\frac{2 \varepsilon(p+2)(1-a)}{\eta+2}\left(\left\|u_{t}\right\|_{\eta+2}^{\eta+2}+\left\|v_{t}\right\|_{\eta+2}^{\eta+2}\right) \\
& +\varepsilon(p+2)(1-a)\left(\left\|\nabla u_{t}\right\|_{2}^{2}+\left\|\nabla v_{t}\right\|_{2}^{2}\right) \\
& +\varepsilon(p+2)(1-a)\left(1-\int_{0}^{t} g(s) d s\right)\|\nabla u\|_{2}^{2} \\
& +\varepsilon(p+2)(1-a)\left(1-\int_{0}^{t} h(s) d s\right)\|\nabla v\|_{2}^{2} \\
& +\varepsilon(p+2)(1-a)\left(\left(h_{1} o \nabla u\right)+\left(h_{2} o \nabla v\right)\right) \\
& +\frac{\varepsilon(p+2)(1-a)}{\gamma+1}\left(\|\nabla u\|_{2}^{2(\gamma+1)}+\|\nabla v\|_{2}^{2(\gamma+1)}\right) \\
& +\varepsilon 2(p+2)(1-a) \mathbb{H}(t) .
\end{aligned}
$$

Substituting in (3.16), and by using (2.3), we get

$$
\begin{aligned}
\mathcal{K}^{\prime}(t) \geq\{ & (1-\alpha)-\varepsilon \kappa\} \mathbb{H}^{-\alpha} \mathbb{H}^{\prime}(t)+\varepsilon\{(p+2)(1-a)+1\}\left(\left\|\nabla u_{t}\right\|_{2}^{2}+\left\|\nabla v_{t}\right\|_{2}^{2}\right) \\
& +\varepsilon\left\{\frac{2 \varepsilon(p+2)(1-a)}{\eta+2}+\frac{1}{\eta+1}\right\}\left(\left\|u_{t}\right\|_{\eta+2}^{\eta+2}+\left\|v_{t}\right\|_{\eta+2}^{\eta+2}\right) \\
& +\varepsilon\left\{(p+2)(1-a)\left(1-\int_{0}^{t} h_{1}(s) d s\right)-\left(1-\frac{1}{2} \int_{0}^{t} h_{1}(s) d s\right)\right\}\|\nabla u\|_{2}^{2} \\
& +\varepsilon\left\{(p+2)(1-a)\left(1-\int_{0}^{t} h_{2}(s) d s\right)-\left(1-\frac{1}{2} \int_{0}^{t} h_{2}(s) d s\right)\right\}\|\nabla v\|_{2}^{2} \\
& +\varepsilon\left\{(p+2)(1-a)-\frac{1}{2}\right\}\left(h_{1} o \nabla u+h_{2} o \nabla v\right) \\
& +\varepsilon\left\{\frac{(p+2)(1-a)}{\gamma+1}-1\right\}\left(\|\nabla u\|_{2}^{2(\gamma+1)}+\|\nabla v\|_{2}^{2(\gamma+1)}\right) \\
& +\varepsilon\left\{c_{0} a-\left(M_{3} C_{1}(\kappa)+M_{4} C_{2}(\kappa)\right)\right\}\left(\|u\|_{2(p+2)}^{2(p+2)}+\|v\|_{2(p+2)}^{2(p+2)}\right) \\
& +\varepsilon\left\{\left(2(p+2)(1-a)-\left(M_{3} C_{1}(\kappa)+M_{4} C_{2}(\kappa)\right)\right\} \mathbb{H}(t)\right.
\end{aligned}
$$

where

$$
\begin{aligned}
& M_{3}:=M_{1}\left(1+\frac{l \delta_{3}^{\left(\frac{l+j+1}{l}\right)}}{l+j+1}+\frac{(j+1) \delta_{3}^{-\left(\frac{l+j+1}{l}\right)}}{l+j+1}\right)>0 . \\
& M_{4}:=M_{2}\left(1+\frac{\varrho \delta_{4}^{\left(\frac{\varrho+s+1}{\varrho}\right)}}{\varrho+s+1}+\frac{(s+1) \delta_{4}^{-\left(\frac{\varrho+s+1}{\varrho}\right)}}{\varrho+s+1}\right)>0 .
\end{aligned}
$$

At this stage, we take $a>0$ small enough so that

$$
(p+2)(1-a)>1+\gamma
$$

we have

$$
\begin{aligned}
& \lambda_{1}:=(p+2)(1-a)-1>0 \\
& \lambda_{2}:=(p+2)(1-a)-\frac{1}{2}>0
\end{aligned}
$$




$$
\lambda_{3}:=\frac{(p+2)(1-a)}{\gamma+1}-1>0
$$

and we assume that

$$
\max \left\{\int_{0}^{\infty} h_{1}(s) d s, \int_{0}^{\infty} h_{2}(s) d s\right\}<\frac{(p+2)(1-a)-1}{\left((p+2)(1-a)-\frac{1}{2}\right)}=\frac{2 \lambda_{1}}{2 \lambda_{1}+1}
$$

gives

$$
\begin{aligned}
& \lambda_{4}=\left\{((p+2)(1-a)-1)-\int_{0}^{t} h_{1}(s) d s\left((p+2)(1-a)-\frac{1}{2}\right)\right\}>0, \\
& \lambda_{5}=\left\{((p+2)(1-a)-1)-\int_{0}^{t} h_{2}(s) d s\left((p+2)(1-a)-\frac{1}{2}\right)\right\}>0,
\end{aligned}
$$

then we choose $\kappa$ so large that

$$
\begin{aligned}
& \lambda_{6}=a c_{0}-\left(M_{3} C_{1}(\kappa)+M_{4} C_{2}(\kappa)\right)>0, \\
& \lambda_{7}=2(p+2)(1-a)-\left(M_{3} C_{1}(\kappa)+M_{4} C_{2}(\kappa)\right)>0 .
\end{aligned}
$$

Finally, we fix $\kappa, a$, and we appoint $\varepsilon$ small enough so that

$$
\lambda_{8}=(1-\alpha)-\varepsilon \kappa>0 .
$$

Thus, for some $\beta>0$, estimate (3.29) becomes

$$
\begin{aligned}
\mathcal{K}^{\prime}(t) \geq & \beta\left\{\mathbb{H}(t)+\left\|u_{t}\right\|_{\eta+2}^{\eta+2}+\left\|v_{t}\right\|_{\eta+2}^{\eta+2}+\left\|\nabla u_{t}\right\|_{2}^{2}+\left\|\nabla v_{t}\right\|_{2}^{2}+\|\nabla u\|_{2}^{2}+\|\nabla v\|_{2}^{2}\right. \\
& \left.+\|\nabla u\|_{2}^{2(\gamma+1)}+\|\nabla v\|_{2}^{2(\gamma+1)}+\left(h_{1} o \nabla u\right)+\left(h_{2} o \nabla v\right)+\|u\|_{2(p+2)}^{2(p+2)}+\|u\|_{2(p+2)}^{2(p+2)}\right\} .
\end{aligned}
$$

By (2.3), for some $\beta_{1}>0$, we obtain

$$
\begin{aligned}
\mathcal{K}^{\prime}(t) \geq & \beta_{1}\left\{\mathbb{H}(t)+\left\|u_{t}\right\|_{\eta+2}^{\eta+2}+\left\|v_{t}\right\|_{\eta+2}^{\eta+2}+\left\|\nabla u_{t}\right\|_{2}^{2}+\left\|\nabla v_{t}\right\|_{2}^{2}+\|\nabla u\|_{2}^{2}\right. \\
& +\|\nabla v\|_{2}^{2}+\|\nabla u\|_{2}^{2(\gamma+1)}+\|\nabla v\|_{2}^{2(\gamma+1)}+\left(h_{1} o \nabla u\right)+\left(h_{2} o \nabla v\right) \\
& \left.+\|u+v\|_{2(p+2)}^{2(p+2)}+2\|u v\|_{p+2}^{p+2}\right\}
\end{aligned}
$$

and

$$
\mathcal{K}(t) \geq \mathcal{K}(0)>0, \quad t>0 .
$$

Next, using Holder's and Young's inequalities, we have

$$
\begin{aligned}
\left|\int_{\Omega}\left(u\left|u_{t}\right|^{\eta} u_{t}+v\left|v_{t}\right|^{\eta} v_{t}\right) d x\right|^{\frac{1}{1-\alpha}} \leq & C\left[\|u\|_{2(p+2)}^{\frac{\theta}{1-\alpha}}+\left\|u_{t}\right\|_{\eta+2}^{\frac{\mu}{1-\alpha}}\right. \\
& \left.+\|v\|_{2(p+2)}^{\frac{\theta}{1-\alpha}}+\left\|v_{t}\right\|_{\eta+2}^{\frac{\mu}{1-\alpha}}\right]
\end{aligned}
$$

where $\frac{1}{\mu}+\frac{1}{\theta}=1$. 
We take $\mu=(\eta+2)(1-\alpha)$ to get

$$
\frac{\theta}{1-\alpha}=\frac{\eta+2}{(1-\alpha)(\eta+2)-1} \leq 2(p+2)
$$

Subsequently, by using (3.8), (3.6), and (3.22), we obtain

$$
\begin{aligned}
& \|u\|_{2(p+2)}^{\frac{\eta+2}{(1-\alpha)(\eta+2)-1}} \leq d\left(\|u\|_{2(p+2)}^{2(p+2)}+\mathbb{H}(t)\right), \\
& \|v\|_{2(p+2)}^{\frac{\eta+2}{(1-\alpha)(\eta+2)-1}} \leq d\left(\|v\|_{2(p+2)}^{2(p+2)}+\mathbb{H}(t)\right), \quad \forall t \geq 0 .
\end{aligned}
$$

Therefore,

$$
\begin{aligned}
& \left|\int_{\Omega}\left(u\left|u_{t}\right|^{\eta} u_{t}+v\left|v_{t}\right|^{\eta} v_{t}\right) d x\right|^{\frac{1}{1-\alpha}} \\
& \quad \leq c_{13}\left\{\|u\|_{2(p+2)}^{2(p+2)}+\|v\|_{2(p+2)}^{2(p+2)}+\left\|u_{t}\right\|_{\eta+2}^{\eta+2}+\left\|v_{t}\right\|_{\eta+2}^{\eta+2}+\mathbb{H}(t)\right\}
\end{aligned}
$$

Similarly, we have

$$
\left|\int_{\Omega}\left(\nabla u \nabla u_{t}+\nabla v \nabla v_{t}\right) d x\right|^{\frac{1}{1-\alpha}} \leq C\left[\|\nabla u\|_{2}^{\frac{\theta}{1-\alpha}}+\left\|\nabla u_{t}\right\|_{2}^{\frac{\mu}{1-\alpha}}+\|\nabla v\|_{2}^{\frac{\theta}{1-\alpha}}+\left\|\nabla v_{t}\right\|_{2}^{\frac{\mu}{1-\alpha}}\right]
$$

where $\frac{1}{\mu}+\frac{1}{\theta}=1$.

We take $\theta=2(\gamma+1)(1-\alpha)$ to get

$$
\begin{aligned}
\frac{\mu}{1-\alpha}=\frac{2(\gamma+1)}{2(1-\alpha)(\gamma+1)-1} \leq & \\
\left|\int_{\Omega}\left(\nabla u \nabla u_{t}+\nabla \nu \nabla v_{t}\right) d x\right|^{\frac{1}{1-\alpha}} & \leq c_{14}\left\{\|\nabla u\|_{2}^{2(\gamma+1)}+\|\nabla v\|_{2}^{2(\gamma+1)}\right. \\
& \left.+\left\|\nabla u_{t}\right\|_{2}^{2}+\left\|\nabla v_{t}\right\|_{2}^{2}\right\} .
\end{aligned}
$$

Hence, by (3.35) and (3.36),

$$
\begin{aligned}
\mathcal{K} \frac{1}{1-\alpha}(t)= & \left(\mathbb{H}^{1-\alpha}+\frac{\varepsilon}{\eta+1} \int_{\Omega}\left(u\left|u_{t}\right|^{\eta} u_{t}+v\left|v_{t}\right|^{\eta} v_{t}\right) d x\right. \\
& \left.+\varepsilon \int_{\Omega}\left(\nabla u_{t} \nabla u+\nabla v_{t} \nabla v\right) d x\right)^{\frac{1}{1-\alpha}} \\
\leq & c\left(\mathbb{H}(t)+\left|\int_{\Omega}\left(u\left|u_{t}\right|^{\eta} u_{t}+v\left|v_{t}\right|^{\eta} v_{t}\right) d x\right|^{\frac{1}{1-\alpha}}+\|\nabla u\|_{2}^{\frac{2}{1-\alpha}}+\|\nabla v\|_{2}^{\frac{2}{1-\alpha}}\right. \\
& \left.+\left\|\nabla u_{t}\right\|_{2}^{\frac{2}{1-\alpha}}+\left\|\nabla v_{t}\right\|_{2}^{\frac{2}{1-\alpha}}\right) \\
\leq & c\left(\mathbb{H}(t)+\left\|u_{t}\right\|_{\eta+2}^{\eta+2}+\left\|v_{t}\right\|_{\eta+2}^{\eta+2}+\|\nabla u\|_{2}^{\gamma+1}+\|\nabla v\|_{2}^{\gamma+1}+\left\|\nabla u_{t}\right\|_{2}^{2}\right. \\
& \left.+\left\|\nabla v_{t}\right\|_{2}^{2}+\left(h_{1} o \nabla u\right)+\left(h_{2} o \nabla v\right)+\|u\|_{2(p+2)}^{2(p+2)}+\|v\|_{2(p+2)}^{2(p+2)}\right) \\
\leq & c\left(\mathbb{H}(t)+\left\|u_{t}\right\|_{\eta+2}^{\eta+2}+\left\|v_{t}\right\|_{\eta+2}^{\eta+2}+\|\nabla u\|_{2}^{\gamma+1}+\|\nabla v\|_{2}^{\gamma+1}+\left\|\nabla u_{t}\right\|_{2}^{2}\right.
\end{aligned}
$$




$$
\begin{aligned}
& +\left\|\nabla v_{t}\right\|_{2}^{2}+\|\nabla u\|_{2}^{2}+\|\nabla v\|_{2}^{2}+\left(h_{1} o \nabla u\right)+\left(h_{2} o \nabla v\right) \\
& \left.+\|u\|_{2(p+2)}^{2(p+2)}+\|v\|_{2(p+2)}^{2(p+2)}\right)
\end{aligned}
$$

From (3.31) and (3.37), it gives

$$
\mathcal{K}^{\prime}(t) \geq \lambda \mathcal{K}^{\frac{1}{1-\alpha}}(t)
$$

where $\lambda>0$, this depends only on $\beta$ and $c$.

By integration of (3.38), we obtain

$$
\mathcal{K}^{\frac{\alpha}{1-\alpha}}(t) \geq \frac{1}{\mathcal{K}^{\frac{-\alpha}{1-\alpha}}(0)-\lambda \frac{\alpha}{(1-\alpha)} t} .
$$

Hence, $\mathcal{K}(t)$ blows up in time

$$
T \leq T^{*}=\frac{1-\alpha}{\lambda \alpha \mathcal{K}^{\alpha /(1-\alpha)}(0)} .
$$

Then the proof is completed.

\section{Conclusion}

In this paper, we are interested in the blow-up for a quasilinear system of viscoelastic equations with degenerate damping and general source terms according to some suitable hypothesis. This work is a general case of the recent results of Boulaaras' works in [11, 21] using the energy method. Next we will prove the result of local existence of this studied problem based on the recent result in [4].

\section{Acknowledgements}

The authors would like to thank the anonymous referees and the handling editor for their careful reading and for relevant remarks/suggestions which helped to improve the paper. The fifth author extends their appreciation to the Deanship of Scientific Research at King Khalid University for funding this work through Research Group Project under Grant no. (R.G.P-2/53/42).

\section{Funding}

Not applicable.

Availability of data and materials Not applicable.

\section{Declarations}

Ethics approval and consent to participate

Not applicable.

Consent for publication

Not applicable.

Competing interests

The authors declare that they have no competing interests.

Authors' contributions

The authors contributed equally in this article. They have all read and approved the final manuscript. 


\section{Author details}

'Department of Mathematics, College of Sciences and Arts, ArRas, Qassim University, Buraydah, Kingdom of Saudi Arabia. ${ }^{2}$ Laboratory of Fundamental and Applied Mathematics of Oran (LMFAO), University of Oran 1, Oran, Algeria. ${ }^{3}$ Department of Mathematics, Faculty of Exact Sciences, University of El Oued, El Oued, Algeria. ${ }^{4}$ Anand International College of Engineering, Near Kanota, Agra Road, Jaipur 303012, Rajasthan, India. ${ }^{5}$ Department of Mathematics, Faculty of Science, King Khalid University, Abha 61471, Saudi Arabia. ${ }^{6}$ Mathematics Department, Faculty of Science, South Valley University, Qena 83523, Egypt. ${ }^{7}$ College of Industrial Engineering, King Khalid University, Abha 61471, Saudi Arabia.

\section{Publisher's Note}

Springer Nature remains neutral with regard to jurisdictional claims in published maps and institutional affiliations.

Received: 17 August 2021 Accepted: 29 September 2021 Published online: 09 October 2021

\section{References}

1. Agre, K., Rammaha, M.A.: Systems of nonlinear wave equations with damping and source terms. Differ. Integral Equ. $19,1235-1270(2007)$

2. Ball, J.: Remarks on blow-up and nonexistence theorems for nonlinear evolution equation. Q. J. Math. 28, 473-486 (1977)

3. Bland, D.R.: The Theory of Linear Viscoelasticity. Courier Dover Publications, Mineola (2016)

4. Boulaaras, S., Choucha, A., Ouchenane, D., Alharbi, A., Abdullah, M.: Result of local existence of solutions of nonlocal viscoelastic system with respect to the nonlinearity of source terms. Fractals 30(1), 2240027 (2022). https://doi.org/10.1142/S0218348X22400278

5. Boulaaras, S., Choucha, A., Ouchenane, D., Cherif, B.: Blow up of solutions of two singular nonlinear viscoelastic equations with general source and localized frictional damping terms. Adv. Differ. Equ. 2020, 310 (2020)

6. Boulaaras, S., Draifia, A., Zennir, K.: General decay of nonlinear viscoelastic Kirchhoff equation with Balakrishnan-Taylor damping and logarithmic nonlinearity. Math. Methods Appl. Sci. 42, 4795-4814 (2019)

7. Cavalcanti, M.M., Cavalcanti, D., Ferreira, J.: Existence and uniform decay for nonlinear viscoelastic equation with strong damping. Math. Methods Appl. Sci. 24, 1043-1053 (2001)

8. Choucha, A., Boulaaras, S., Ouchenane, D., Beloul, S.: General decay of nonlinear viscoelastic Kirchhoff equation with Balakrishnan-Taylor damping, logarithmic nonlinearity and distributed delay terms. Math. Methods Appl. Sci. 44, 5436-5457 (2021). https://doi.org/10.1002/mma.7121

9. Choucha, A., Boulaaras, S.M., Ouchenane, D., Cherif, B.B., Abdalla, M.: Exponential stability of swelling porous elastic with a viscoelastic damping and distributed delay term. J. Funct. Spaces 2021, Article ID 5581634 (2021). https://doi.org/10.1155/2021/5581634

10. Choucha, A., Ouchenane, D., Boulaaras, S.: Well posedness and stability result for a thermoelastic laminated Timoshenko beam with distributed delay term. Math. Methods Appl. Sci. 43, 9983-10004 (2020). https://doi.org/10.1002/mma.6673

11. Choucha, A., Ouchenane, D., Boulaaras, S.: Blow-up of a nonlinear viscoelastic wave equation with distributed delay combined with strong damping and source terms. J. Nonlinear Funct. Anal. 2020, Article ID 31 (2020). https://doi.org/10.23952/jnfa.2020.31

12. Choucha, A., Ouchenane, D., Zennir, K., Feng, B.: Global well-posedness and exponential stability results of a class of Bresse-Timoshenko-type systems with distributed delay term. Math. Methods Appl. Sci., 1-26 (2020). https://doi.org/10.1002/mma.6437

13. Coleman, B.D., Noll, W.: Foundations of linear viscoelasticity. Rev. Mod. Phys. 33(2), 239 (1961)

14. Ekinci, F., Piskin, E., Boulaaras, S.M., Mekawy, I.: Global existence and general decay of solutions for a quasilinear system with degenerate damping terms. J. Funct. Spaces (2021)

15. Georgiev, $V_{\text {., }}$ Todorova, G.: Existence of a solution of the wave equation with nonlinear damping and source term. J. Differ. Equ. 109, 295-308 (1994)

16. He, L.: On decay and blow-up of solutions for a system of equations. Appl. Anal. 100(11), 2449-2477 (2021). https://doi.org/10.1080/00036811.2019.1689562

17. Kirchhoff, G.: Vorlesungen uber Mechanik. Tauber, Leipzig (1883)

18. Liang, G., Zhaoqin, Y., Guonguang, L.: Blow up and global existence for a nonlinear viscoelastic wave equation with strong damping and nonlinear damping and source terms. Appl. Math. 6, 806-816 (2015)

19. Liu, W.: General decay and blow-up of solution for a quasilinear viscoelastic problem with nonlinear source. Nonlinear Anal. 73, 1890-1904 (2010)

20. Mesloub, F., Boulaaras, S.: General decay for a viscoelastic problem with not necessarily decreasing kernel. J. Appl. Math. Comput. 58, 647-665 (2018). https://doi.org/10.1007/S12190-017-1161-9

21. Mezouar, N., Boulaaras, S.: Global existence and decay of solutions for a class of viscoelastic Kirchhoff equation. Bull. Malays. Math. Sci. Soc. 43, 725-755 (2020)

22. Mezouar, N., Boulaaras, S.: Global existence of solutions to a viscoelastic non-degenerate Kirchhoff equation. Appl. Anal. 99, 1724-1748 (2020)

23. Ouchenane, D., Boulaaras, S., Mesloub, F.: General decay for a viscoelastic problem with not necessarily decreasing kernel. Appl. Anal. https://doi.org/10.1080/00036811.2018

24. Piskin, E., Ekinci, F.: General decay and blowup of solutions for coupled viscoelastic equation of Kirchhoff type with degenerate damping terms. Math. Methods Appl. Sci. 42(16), 5468-5488 (2019)

25. Song, H.T., Xue, D.S.: Blow up in a nonlinear viscoelastic wave equation with strong damping. Nonlinear Anal. 109, 245-251 (2014). https://doi.org/10.1016/j.na.2014.06.012

26. Song, H.T., Zhong, C.K.: Blow-up of solutions of a nonlinear viscoelastic wave equation. Nonlinear Anal., Real World Appl. 11, 3877-3883 (2010). https://doi.org/10.1016/j.nonrwa.2010.02.015

27. Wu, S.T.: General decay of energy for a viscoelastic equation with damping and source terms. Taiwan. J. Math. 16(1), $113-128$ (2012)

28. Yang, H., Fang, S., Liang, F., Li, M.: A general stability result for second order stochastic quasi-linear evolution equations with memory. Bound. Value Probl. 62, 1-16 (2020) 